\title{
The best single-observational and two-observational percentile estimations in the exponentiated Weibull-geometric distribution compared with maximum likelihood and percentile estimations
}

\author{
Shahram Yaghoobzadeh Shahrastani ${ }^{1}$ (D) Masoud Yarmohammadi ${ }^{1}$
}

Received: 25 May 2017/Revised: 30 November 2017/Published online: 15 September 2018

(C) The Author(s) 2018

\begin{abstract}
In this research the best single-observation percentile estimation (BSPE) and best two-observation percentile estimation (BTPE), are introduced. Then theses estimators are obtained for probability density function and cumulative distribution function of the exponentiated Weibull-geometric (EWG) with increasing, decreasing, bathtub and unimodal shaped failure rate function. Finally, these estimators are compared with the maximum likelihood (ML) and percentile (PC) estimations using the Monte Carlo simulation and a real data set.
\end{abstract}

Keywords Single-observational percentile estimation . Two-observational percentile estimation - Maximum likelihood estimation · Percentile estimation · Monte Carlo simulation

\section{Introduction}

The estimation of probability density function (PDF) and cumulative density function (CDF) of several lifetime distributions using the maximum likelihood (ML), uniformly minimum variance unbiased (UMVU), percentile (PC), least squares (LS) and weighted least squares (WLS) estimators have been obtained and compared by researchers. A number of papers have been attempted to estimate the lifetime distribution parameters, for instance the estimation of pdf and cdf of the Pareto distribution by Dixit and Jabbari Nooghabi (2010), exponentiated Pareto

Shahram Yaghoobzadeh Shahrastani

yagoubzade@gmail.com

1 Department of Statistics, Payame Noor University, P. O. Box 19395-4697, Tehran, Islamic Republic of Iran distribution by Jabbari Nooghabi and Jabbari Nooghabi (2010), exponentiated Gumbel distribution by Bagheri et al. (2013b), generalized Rayliegh distribution by Alizadeh et al. (2013) and generalized Poisson-exponential distribution by Bagheri et al. (2013a). Note that Menon (1963) and Zanakis and Mann (1982) estimated the parameters of Weibull distribution by best single-observation percentile estimation (BSPE) and best two-observation percentile estimation (BTPE), but in this research the PDF and CDF of the Exponentiated Weibull-Geometric (EWG) which is originally introduced by Mahmoudi and Shiran (2012) are obtained by BSPE and BTPE methods for One or Two known parameters and compared with the corresponding estimations found by PC and MLE procedures.

According to the structure in this paper, in Sects. 2 and 3, the BEPE, PCE, MLE and BTPE, PCE, MLE are obtained respectively. By using the Monte Carlo simulations, estimators were compared in Sect. 4, and the results for real data are provided in Sect. 5.

\section{Calculating estimations when only one parameter is unknown}

Let $X_{1}, \ldots, X_{n}$ is a random sample with ordinal statistics of $Y_{1}, \ldots, Y_{n}$, of a distribution with the following probability density and cumulative distribution functions:

$$
f(x ; \alpha, \beta, \gamma, \theta)=\frac{\alpha \beta^{\gamma} \gamma(1-\theta) x^{\gamma-1} e^{-(\beta x)^{\gamma}}\left(1-e^{-(\beta x)^{\gamma}}\right)^{\alpha-1}}{\left[1-\theta\left(1-e^{-(\beta x)^{\gamma}}\right)^{\alpha}\right]^{2}}
$$


$F(x ; \alpha, \beta, \gamma, \theta)=\frac{(1-\theta)\left(1-e^{-(\beta x)^{\gamma}}\right)^{\alpha}}{1-\theta\left(1-e^{-(\beta x)^{\gamma}}\right)^{\alpha}}$

Such that $x>0, \alpha>0, \beta>0, \gamma>0,0<\theta<1$. In this section, assuming that parameters $\beta, \gamma, \theta$ are known and parameter $\alpha$ is unknown, the BSPE, PCE and MLE of $\alpha$ are obtained.

\subsection{Estimation of the BSP}

If $Y_{k}$ is the $p$-th percentile $(0<p<1)$ of distribution (2), then

$p=F\left(Y_{k} ; \alpha, \beta, \gamma, \theta\right)=\frac{(1-\theta)\left(1-e^{-\left(\beta Y_{k}\right)^{\gamma}}\right)^{\alpha}}{1-\theta\left(1-e^{-\left(\beta Y_{k}\right)^{\gamma}}\right)^{\alpha}}$

where $k=[n p]$, if $n p$ is an integer, otherwise, $k=[n p]+1$ where $[n p]$ is the greatest integer smaller than $n p$. Therefore, a single-observation percentile estimation of $\alpha$ which is shown by $\alpha^{*}$ is as follows:

$\alpha^{*}=\frac{\log \frac{p}{1-\theta(1-p)}}{\log \left(1-e^{\left(-\beta Y_{k}\right)^{\gamma}}\right)}=\frac{\log \left[-\log \left(1-p^{*}\right)\right]}{\log Z_{k}}$

Such that $p^{*}=1-e^{-\frac{p}{1-\theta(1-p)}}$ and $\quad Z_{k}=1-e^{\left(-\beta Y_{k}\right)^{\gamma}}$. According to Dubey (1967, p. 122), $\alpha^{*}$ has an asymptotic normal distribution with mean of $\alpha$ and variance of

$$
\begin{aligned}
\operatorname{Var}\left(\alpha^{*}\right) & =\frac{\alpha^{2} p^{*}}{n\left(1-p^{*}\right) \log ^{2}\left(1-p^{*}\right) \log ^{2}\left[-\log \left(1-p^{*}\right)\right]} \\
& =\frac{\alpha^{2}\left(1-e^{-q}\right)}{n q^{2} e^{-q} \log ^{2} q}
\end{aligned}
$$

where $q=\frac{p}{1-\theta(1-p)}$. Now $q$ is determined in a way that $\operatorname{Var}\left(\alpha^{*}\right)$ is minimum, which in this case, solves the equation

$q \log q-2(1+\log q)\left(1+e^{-q}\right)=0$

By an iterative method, $q=0.1189$ and finally, the optimal $p$ is obtained by the following relation.

$p=\frac{0.1189(1-\theta)}{1-0.1189 \theta}$

Therefore, the BSPE of $\alpha$ as shown by $\hat{\alpha}_{B S P E}$ is determined as follows:

$\hat{\alpha}_{B S P E}=\frac{\log [0.1189(1-\theta) /(1-0.1189 \theta)]}{\log \left(1-e^{-\left(\beta Y_{k}\right)^{\gamma}}\right)}$

Such that, $k=\left[n \frac{0.1189(1-\theta)}{1-0.1189 \theta}\right]$ or $k=1+\left[n \frac{0.1189(1-\theta)}{1-0.1189 \theta}\right]$.

Thus, the BSPE of functions (1) and (2) are obtained by the following relation, respectively.

$$
\begin{aligned}
& \hat{f}_{B S P E}(x ; \alpha, \beta, \gamma, \theta) \\
& =\frac{\hat{\alpha}_{B S P E} \beta^{\gamma} \gamma(1-\theta) x^{\gamma-1} e^{-(\beta x)^{\gamma}}\left(1-e^{-(\beta x)^{\gamma}}\right)^{\left(\hat{\alpha}_{B S P E}\right)-1}}{\left[1-\theta\left(1-e^{-(\beta x)^{\gamma}}\right)^{\hat{\alpha}_{B S P E}}\right]^{2}} \\
& \hat{F}_{B S P E}(x ; \alpha, \beta, \gamma, \theta)=\frac{(1-\theta)\left(1-e^{-(\beta x)^{\gamma}}\right)^{\hat{\alpha}_{B S P E}}}{1-\theta\left(1-e^{\left.-(\beta x)^{\gamma}\right)^{\hat{\beta}_{B S P E}}}\right.}
\end{aligned}
$$

\subsection{PCE}

Let $X_{1}, \ldots, X_{n}$ is a random sample distribution with CDF given in (2) with order statistics of $Y_{1}, \ldots, Y_{n}$, and $p_{i}$ is the percentile of $Y_{i}$, then, $F\left(Y_{i}, \alpha, \beta, \gamma, \theta\right)=p_{i}$ or

$\log \frac{p_{i}}{\left[1-\theta\left(1-p_{i}\right)\right]}=\alpha \log \left(1-e^{-\left(\beta Y_{i}\right)^{\gamma}}\right)$

The PCE of $\alpha$ which is shown by $\hat{\alpha}_{P C E}$ is obtained by the minimization of

$\sum_{i=1}^{n}\left[\alpha \log \left(1-e^{-\left(\beta Y_{i}\right)^{\gamma}}\right)-\log \frac{p_{i}}{\left[1-\theta\left(1-p_{i}\right)\right]}\right]^{2}$

with respect to $\alpha$. $\left(p_{i}=\frac{i}{n+1}\right)$, so

$\hat{\alpha}_{P C E}=\sum_{i=1}^{n} \log \frac{p_{i}\left(1-e^{-\left(\beta Y_{i}\right)^{\gamma}}\right)}{\left[1-\theta\left(1-p_{i}\right)\right]} / \sum_{i=1}^{n}\left(1-e^{-\left(\beta Y_{i}\right)^{\gamma}}\right)^{2}$

Therefore, the PCEs of functions (1) and (2) are obtained as follows:

$$
\begin{aligned}
& \hat{f}_{P C E}(x ; \alpha, \beta, \gamma, \theta) \\
& =\frac{\hat{\alpha}_{P C E} \beta^{\gamma} \gamma(1-\theta) x^{\gamma-1} e^{-(\beta x)^{\gamma}}\left(1-e^{-(\beta x)^{\gamma}}\right)^{\left(\hat{\alpha}_{P C E}\right)-1}}{\left[1-\theta\left(1-e^{-(\beta x)^{\gamma}}\right)^{\hat{\alpha}_{P C E}}\right]^{2}} \\
& \hat{F}_{P C E}(x ; \alpha, \beta, \gamma, \theta)=\frac{(1-\theta)\left(1-e^{-(\beta x)^{\gamma}}\right)^{\hat{\alpha}_{P C E}}}{1-\theta\left(1-e^{-(\beta x)^{\gamma}}\right)^{\hat{\alpha}_{P C E}}}
\end{aligned}
$$

For more details about the PCE method, see Kao $(1958,1959)$ and Johnson et al. (1994). Mean square error (MSE) of percentile estimations of functions (1) and (2) is calculated by Monte Carlo simulation method of the sample mean.

\subsection{MLE}

According to a random sample of $X_{1}, \ldots, X_{n}$ of distribution with the probability density function (1), the MLE of the parameter $\alpha$, i.e. $\hat{\alpha}_{M L E}$ is obtained by:

$\frac{n}{\alpha}+\sum_{i=1}^{n} \frac{\left[1+\theta\left(1-e^{-\left(\beta x_{i}\right)^{\gamma}}\right)^{\alpha}\right] \log \left(1-e^{-\left(\beta x_{i}\right)^{\gamma}}\right)}{1-\theta\left(1-e^{-\left(\beta x_{i}\right)^{\gamma}}\right)^{\alpha}}=0$ 
where replacing $\hat{\alpha}_{M L E}$ by $\alpha$ in relations (1) and (2), The MLE of the probability density and cumulative distribution functions of EWG distribution can be obtained. Moreover, by Monte Carlo simulation method of the sample mean, the mean square error (MSE) of the MLE of functions (1) and (2) could be found.

\section{Calculating estimators when two parameters are unknown}

In this section, a random sample of size $n$ from the pdf given in (1) is considered. We assume that the parameters $\gamma$ and $\beta$ are unknown, and parameters $\alpha$ and $\theta$ are known,. Then the BTPE, PCE and MLE of $\gamma$ and $\beta$, for the pdf (1) and cdf (2) are obtained.

\subsection{BTPE}

Suppose $X_{1}, \ldots, X_{n}$ is a random sample of distribution with cdf (2) with ordinal statistics of $Y_{1}, \ldots, Y_{n}$, and $p_{i}$ is the percentile of $Y_{i}$, then, $F\left(Y_{i}, \alpha, \beta, \gamma, \theta\right)=p_{i}$ or

$\gamma\left(\log \beta+\log Y_{i}\right)=\log \left\{-\log \left[1-\left(\frac{p_{i}}{1-\theta+\theta p_{i}}\right)^{\frac{1}{\alpha}}\right]\right\}$

such that for two real values of $p_{1}$ and $p_{2}\left(0<p_{1}<p_{2}<1\right)$ and with the help of relation (4), a two-observational percentile estimation of $\gamma$ which is shown by $\gamma^{*}$ can be obtained as follows:

$$
\begin{aligned}
\gamma^{*} & =\frac{\log \left\{-\log \left[1-\left(\frac{p_{1}}{1-\theta+\theta p_{1}}\right)^{\frac{1}{\alpha}}\right]\right\}-\log \left\{-\log \left[1-\left(\frac{p_{2}}{1-\theta+\theta p_{2}}\right)^{\frac{1}{\alpha}}\right]\right\}}{\log Y_{k_{1}}-\log Y_{k_{2}}} \\
& =\frac{\log \left[-\log \left(1-p_{1}^{*}\right)\right]-\log \left[-\log \left(1-p_{2}^{*}\right)\right]}{\log Y_{k_{1}}-\log Y_{k_{2}}}=\frac{k}{\log Y_{k_{1}}-\log Y_{k_{2}}}
\end{aligned}
$$

where

$k=\log \left[-\log \left(1-p_{1}^{*}\right)\right]-\log \left[-\log \left(1-p_{2}^{*}\right)\right]$

and for $i=1,2, k_{i}=\left[n p_{i}\right]$ or $k_{i}=\left[n p_{i}\right]+1$ and

$p_{i}^{*}=\left(\frac{p_{i}}{1-\theta+\theta p_{i}}\right)^{\frac{1}{\alpha}}$

According to Dubey (1967, p. 122), $\gamma^{*}$ has an asymptotic normal distribution with a mean of $\gamma$ and variance of

$$
\begin{aligned}
& \operatorname{Var}\left(\gamma^{*}\right) \\
& =\frac{\gamma^{2}}{n k^{2}}\left[\frac{p_{1}^{*}}{\left(1-p_{1}^{*}\right) \log ^{2}\left(1-p_{1}^{*}\right)}+\frac{p_{2}^{*}}{\left(1-p_{2}^{*}\right) \log ^{2}\left(1-p_{2}^{*}\right)}\right. \\
& \left.-\frac{2 p_{1}^{*} p_{2}^{*}}{\left(1-p_{1}^{*}\right)\left(1-p_{2}^{*}\right) \log \left(1-p_{1}^{*}\right) \log \left(1-p_{2}^{*}\right)}\right]
\end{aligned}
$$

Now, $p_{1}^{*}$ and $p_{2}^{*}$ should be determined in a way that $\operatorname{Var}\left(\gamma^{*}\right)$ is minimized where, according to Dubey (1967, p. 122), $p_{1}^{*}=0.16730679$ and $p_{2}^{*}=0.97366352$. Therefore, calculating $p_{1}$ and $p_{2}$ with the help of (5), the BTPE of $\gamma$ which is shown by $\hat{\gamma}_{B T P E}$ is obtained as follows:

$\hat{\gamma}_{B T P E}=\frac{\log \left\{-\log \left[1-\left(\frac{q_{1}}{1-\theta+\theta q_{1}}\right)^{\frac{1}{\gamma}}\right]\right\}-\log \left\{-\log \left[1-\left(\frac{q_{2}}{1-\theta+\theta q_{2}}\right)^{\frac{1}{\gamma}}\right]\right\}}{\log Y_{k_{1}}-\log Y_{k_{2}}}$

where

$$
\begin{gathered}
q_{1}=\frac{(1-\theta)(0.16730679)^{\alpha}}{1-\theta(0.16730679)^{\alpha}}, \\
q_{2}=\frac{(1-\theta)(0.97366352)^{\alpha}}{1-\theta(0.97366352)^{\alpha}}
\end{gathered}
$$

In addition, for $p_{1}$ and $p_{2}\left(0<p_{1}<p_{2}<1\right)$, with the help of (3), a TPE of $\beta$ which is shown by $\beta^{*}$ is obtained as follows:

$\beta^{*}=\exp \left(w_{1} \log Y_{k 1}+w_{2} \log Y_{k 2}\right)$

where $w_{1}=T_{2} /\left(T_{1}-T_{2}\right), w_{1}+w_{2}=-1$ and

$T_{i}=\log \left\{-\log \left[1-\left(\frac{p_{i}}{1-\theta+\theta p_{i}}\right)^{\frac{1}{\alpha}}\right]\right\}, \quad i=1,2$

According to Dubey (1967, p. 122), $\beta^{*}$ has an asymptotic normal distribution with a mean of $\beta$ and variance of $\operatorname{Var}\left(\beta^{*}\right)=\frac{\beta^{2}}{n \gamma^{2} k^{2}}\left\{r_{1}^{*}\left(\frac{k-\log k_{1}}{k_{1}}\right)\left[\frac{k-\log k_{1}}{k_{1}}+\frac{2 \log k_{1}}{k_{2}}\right]+\frac{r_{2}^{*} \log ^{2} k_{1}}{k_{2}^{2}}\right\}$

where

$$
\begin{aligned}
k= & \log \left\{-\log \left[1-\left(\frac{p_{1}}{1-\theta+\theta p_{1}}\right)^{\frac{1}{\alpha}}\right]\right\} \\
& -\log \left\{-\log \left[1-\left(\frac{p_{2}}{1-\theta+\theta p_{2}}\right)^{\frac{1}{\alpha}}\right]\right\}
\end{aligned}
$$

And for $i=1,2$

$r_{i}=\left(\frac{p_{i}}{1-\theta+\theta p_{i}}\right)^{\frac{1}{\alpha}}, r_{i}^{*}=\frac{r_{i}}{1-r_{i}}, k_{i}=-\log \left(1-r_{i}\right)$

Now, $r_{1}$ and $r_{2}$ should be determined in a way that $\operatorname{Var}\left(\beta^{*}\right)$ is minimized where, according to Dubey (1967, p. 122), $\quad r_{1}=0.39777$ and $r_{2}=0.82111$. Therefore, 
calculating $p_{1}$ and $p_{2}$ with the help of (6), the BTPE of $\beta$ which is shown by $\hat{\beta}_{B T P E}$ is obtained as follows:

$\hat{\beta}_{B T P E}=\exp \left(\hat{w}_{1} \log Y_{k 1}+\hat{w}_{2} \log Y_{k 2}\right)$

where

$$
\begin{aligned}
& \hat{w}_{1}=\frac{\log \left\{-\log \left[1-\left(\frac{r_{2}^{* *}}{1-\theta+\theta r_{2}^{* * *}}\right)^{\frac{1}{\alpha}}\right]\right\}}{\log \left\{-\log \left[1-\left(\frac{r_{1}^{* *}}{1-\theta+\theta r_{1}^{* *}}\right)^{\frac{1}{\alpha}}\right]\right\}-\log \left\{-\log \left[1-\left(\frac{r_{2}^{* *}}{1-\theta+\theta r_{2}^{* *}}\right)^{\frac{1}{\alpha}}\right]\right\}} \\
& \hat{w}_{2}=\frac{\log \left\{-\log \left[1-\left(\frac{r_{1}^{* *}}{1-\theta+\theta r_{1}^{* * *}}\right)^{\frac{1}{\alpha}}\right]\right\}}{\log \left\{-\log \left[1-\left(\frac{r_{1}^{* *}}{1-\theta+\theta r_{1}^{* * *}}\right)^{\frac{1}{\alpha}}\right]\right\}-\log \left\{-\log \left[1-\left(\frac{r_{2}^{* *}}{1-\theta+\theta r_{2}^{* *}}\right)^{\frac{1}{\alpha}}\right]\right\}}
\end{aligned}
$$

and

$$
r_{1}^{* *}=\frac{(1-\theta)(0.39777)^{\alpha}}{1-\theta(0.39777)^{\alpha}}, \quad r_{2}^{* *}=\frac{(1-\theta)(0.82111)^{\alpha}}{1-\theta(0.82111)^{\alpha}}
$$

where replacing $\hat{\gamma}_{B T P E}$ and $\hat{\beta}_{B T P E}$ in relations (1) and (2), the BTPE, for the pdf (1) and cdf (2), and MSE of these estimators can be achieved.

\subsection{PCE}

Let $X_{1}, \ldots, X_{n}$ is a random sample of distribution with cdf (2) with ordinal statistics of $Y_{1}, \ldots, Y_{n}$, and $p_{i}$ is the percentile of $Y_{i}$, then, $F\left(Y_{i}, \alpha, \beta, \gamma, \theta\right)=p_{i}$ or

$\gamma \log \left(\beta Y_{i}\right)=\log \left\{-\log \left[1-\left(\frac{p_{i}}{1-\theta+\theta p_{i}}\right)^{\frac{1}{\alpha}}\right]\right\}$

Percentile estimations of $\gamma$ and $\beta$ which are shown by $\hat{\gamma}_{P C E}$ and $\hat{\beta}_{P C E}$, respectively, are obtained by minimizing

$$
\left[\left[\sum_{i=1}^{n} \gamma \log \left(\beta Y_{i}\right)-\log \left\{-\log \left[1-\left(\frac{p_{i}}{1-\theta+\theta p_{i}}\right)^{\frac{1}{\alpha}}\right]\right\}^{2}\right]\right]
$$

with respect to $\gamma$ and $\beta$, i.e. by considering the following equations and the Newton-Raphson numerical method are obtained.

$$
\begin{aligned}
& \gamma \sum_{i=1}^{n}\left[\log \left(\beta Y_{i}\right)\right]^{2}-\sum_{i=1}^{n} \log \left(\beta Y_{i}\right) \log \left\{-\log \left[1-\left(\frac{p_{i}}{1-\theta+\theta p_{i}}\right)^{\frac{1}{\alpha}}\right]\right\}=0 \\
& n \gamma \log \beta+\gamma \sum_{i=1}^{n} \log Y_{i} \\
& \quad-\sum_{i=1}^{n} \log \left\{-\log \left[1-\left(\frac{p_{i}}{1-\theta+\theta p_{i}}\right)^{\frac{1}{\alpha}}\right]\right\}=0
\end{aligned}
$$

Replacing $\hat{\gamma}_{M L E}$ and $\hat{\beta}_{B P T E}$ by $\gamma$ and $\beta$ in relations (1) and (2), the PCE of pdf and cdf of EWG distribution, and MSE of these estimators are obtained.

\subsection{MLE}

In this section, according to a random sample of $X_{1}, \ldots, X_{n}$ from a distribution with pdf (1), the MLE of the parameters of $\gamma$ and $\beta$ which are shown by $\hat{\gamma}_{M L E}$ and $\hat{\beta}_{M L E}$, respectively, are obtained by the help of a set of equations

$$
\begin{aligned}
\frac{n}{\gamma} & +\sum_{i=1}^{n}\left[1-\left(\beta x_{i}\right)^{\gamma}\right] \log \left(\beta x_{i}\right) \\
& +(\alpha-1) \sum_{i=1}^{n} \frac{\left(\beta x_{i}\right)^{\gamma} \log \left(\beta x_{i}\right) e^{-\left(\beta x_{i}\right)^{\gamma}}}{1-e^{-\left(\beta x_{i}\right)^{\gamma}}} \\
& +2 \alpha \theta \sum_{i=1}^{n} \frac{\left(\beta x_{i}\right)^{\gamma} \log \left(\beta x_{i}\right) e^{-\left(\beta x_{i}\right)^{\gamma}}\left(1-e^{-\left(\beta x_{i}\right)^{\gamma}}\right)^{\alpha-1}}{\left[1-\theta\left(1-e^{-\left(\beta x_{i}\right)^{\gamma}}\right)^{\alpha}\right]^{2}} \\
& =0
\end{aligned}
$$

$$
\begin{aligned}
n & +\beta^{\gamma}\left\{\sum_{i=1}^{n} x_{i}^{\gamma}+(\alpha-1) \sum_{i=1}^{n} \frac{x_{i}^{\gamma} e^{-\left(\beta x_{i}\right)^{\gamma}}}{1-e^{-\left(\beta x_{i}\right)^{\gamma}}}\right. \\
& \left.+2 \alpha \theta \sum_{i=1}^{n} \frac{x_{i}^{\gamma} e^{-\left(\beta x_{i}\right)^{\gamma}}\left(1-e^{-\left(\beta x_{i}\right)^{\gamma}}\right)^{\alpha-1}}{\left[1-\theta\left(1-e^{-\left(\beta x_{i}\right)^{\gamma}}\right)^{\alpha}\right]^{2}}\right\}=0
\end{aligned}
$$

and the Newton-Raphson numerical method. By replacing the $\gamma$ and $\beta$ by $\hat{\gamma}_{M L E}$ and $\hat{\beta}_{B P T E}$ in relations (1) and (2), the MLE of pdf and cdf of EWG distribution, and MSE of these estimators can be found.

\section{Numerical experiments}

In this section, a Monte Carlo simulation and a numerical example are presented to illustrate all the estimation methods described in the preceding sections.

\subsection{Simulation studies}

In this section, in the first step, using

$X=\frac{1}{\beta}\left\{-\log \left[1-\left(\frac{U}{1-\theta(1-U)}\right)^{\frac{1}{\alpha}}\right]\right\}^{\frac{1}{\gamma}}$

where $U$ has uniformly distribution in the interval $(0,1)$, and for $\alpha=1.5,2,4, \beta=0.25,1.5,3,3.5, \gamma=2,3,4,4.5$ and $\theta=0.2,0.5,0.6,0.8$ random samples are generated as $n=100,200, \ldots, 500$. In the second step, the BSPE, PCE, and MLE of parameter $\alpha$ discussd in Sect. 2, and the BTPE, PCE, and MLE of parameters $\gamma$ and $\beta$ given in Sect. 3 are obtained. In the third step, the mean square error of estimations of functions (1) and (2) is calculated. Steps 1 to 3 were repeated 5000 times and the mean of MSE is obtained from 5000 times repetition was found. The optimal estimator is that one with a smallest Mean MSE. Comparing the results of simulations studies in 
Table 1 A parameter $\alpha$ estimation and estimate the average mean square error (AM) of function (2) the second part of the estimation methods based on simulation results for different values $(\alpha, \beta, \gamma, \theta)$ of EWG distribution

\begin{tabular}{llllllll}
\hline$(\alpha, \beta, \gamma, \theta)$ & $n$ & $\hat{\alpha}_{M L E}$ & $\hat{\alpha}_{P C E}$ & $\hat{\alpha}_{B P S E}$ & $A M\left(\hat{F}_{M L E}\right)$ & $A M\left(\hat{F}_{P C E}\right)$ & $A M\left(\hat{F}_{B P S E}\right)$ \\
\hline$(2,3,4.5,0.6)$ & 100 & 0.72882 & 3.18157 & 2.07177 & 0.043671 & 0.009191 & 0.000345 \\
& 200 & 0.99002 & 3.17151 & 2.02393 & 0.044508 & 0.007156 & 0.000175 \\
& 300 & 0.61051 & 2.80171 & 2.04363 & 0.043832 & 0.006584 & 0.000112 \\
& 400 & 0.84104 & 2.51407 & 2.04793 & 0.043577 & 0.006370 & 0.000086 \\
$(4,1.5,3,0.8)$ & 500 & 0.72915 & 2.79879 & 2.00003 & 0.043471 & 0.006579 & 0.000061 \\
& 100 & 1.77625 & 1.16928 & 4.45912 & 0.063122 & 0.021581 & 0.000207 \\
& 200 & 1.22447 & 7.56527 & 3.94682 & 0.056789 & 0.014676 & 0.000112 \\
$(1.5,0.25,2,0.2)$ & 100 & 0.73831 & 1.85071 & 1.75892 & 0.035794 & 0.002485 & 0.000602 \\
& 300 & 1.46708 & 8.34581 & 4.04442 & 0.055679 & 0.014432 & 0.000073 \\
& 400 & 0.89767 & 5.67570 & 3.87779 & 0.056952 & 0.014692 & 0.000054 \\
& 500 & 1.12513 & 7.47266 & 3.90375 & 0.055481 & 0.012984 & 0.000042 \\
& 300 & 0.74349 & 1.78249 & 1.67786 & 0.035266 & 0.001221 & 0.000292 \\
& 0.65627 & 1.61530 & 1.52227 & 0.035939 & 0.001031 & 0.000196 \\
& 400 & 0.63688 & 1.75294 & 1.56881 & 0.035619 & 0.000965 & 0.000147 \\
& 500 & 0.74216 & 1.70336 & 1.58335 & 0.035433 & 0.000911 & 0.000119 \\
& 100 & 0.60068 & 2.37787 & 1.89153 & 0.041364 & 0.006139 & 0.000422 \\
& 200 & 0.79001 & 2.55847 & 1.93271 & 0.040709 & 0.005613 & 0.000207 \\
& 300 & 0.70204 & 2.50779 & 1.95416 & 0.040697 & 0.004734 & 0.000136 \\
& 400 & 0.87352 & 2.54511 & 1.98047 & 0.040889 & 0.004315 & 0.000103 \\
& 500 & 0.85687 & 2.71180 & 2.04269 & 0.040301 & 0.004426 & 0.000079 \\
\hline
\end{tabular}

\begin{tabular}{llllllll}
\hline$(\alpha, \beta, \gamma, \theta)$ & $n$ & $\hat{\alpha}_{M L E}$ & $\hat{\alpha}_{P C E}$ & $\hat{\alpha}_{B P S E}$ & $A M\left(\hat{f}_{M L E}\right)$ & $A M\left(\hat{f}_{P C E}\right)$ & $A M\left(\hat{f}_{B P S E}\right)$ \\
\hline$(2,3,4.5,0.6)$ & 100 & 0.72882 & 3.18157 & 2.07177 & 1.06530 & 2.11166 & 0.128081 \\
& 200 & 0.99002 & 3.17151 & 2.02393 & 1.04294 & 2.01515 & 0.118559 \\
& 300 & 0.61051 & 2.80171 & 2.04363 & 1.05483 & 2.01050 & 0.114069 \\
& 400 & 0.84104 & 2.51407 & 2.04793 & 1.04966 & 1.99166 & 0.111187 \\
$(4,1.5,3,0.8)$ & 500 & 0.72915 & 2.79879 & 2.00003 & 1.03595 & 2.00381 & 0.108477 \\
& 100 & 1.77625 & 1.16928 & 4.45912 & 0.05557 & 0.122678 & 0.004562 \\
& 200 & 1.22447 & 7.56527 & 3.94682 & 0.05570 & 0.109756 & 0.003513 \\
& 300 & 1.46708 & 8.34581 & 4.04442 & 0.05501 & 0.109418 & 0.003061 \\
$(1.5,0.25,2,0.2)$ & 100 & 0.73831 & 1.85071 & 1.75892 & 0.00116 & 0.001049 & 0.000526 \\
& 400 & 0.89767 & 5.67570 & 3.87779 & 0.05504 & 0.109172 & 0.002886 \\
& 500 & 1.12513 & 7.47266 & 3.90375 & 0.05509 & 0.106254 & 0.002731 \\
& 300 & 0.74349 & 1.78249 & 1.67786 & 0.00113 & 0.000801 & 0.000480 \\
& 0.65627 & 1.61530 & 1.52227 & 0.00112 & 0.000819 & 0.000474 \\
& 400 & 0.63688 & 1.75294 & 1.56881 & 0.00111 & 0.000818 & 0.000469 \\
& 500 & 0.74216 & 1.70336 & 1.58335 & 0.00111 & 0.000819 & 0.000465 \\
$(2,3.5,4,0.5)$ & 100 & 0.60068 & 2.37787 & 1.89153 & 2.38466 & 1.29901 & 0.193829 \\
& 200 & 0.79001 & 2.55847 & 1.93271 & 2.40927 & 1.28597 & 0.178659 \\
& 300 & 0.70204 & 2.50779 & 1.95416 & 2.30379 & 1.27271 & 0.167009 \\
& 400 & 0.87352 & 2.54511 & 1.98047 & 2.25761 & 1.26465 & 0.168395 \\
& 500 & 0.85687 & 2.71180 & 2.04269 & 2.27999 & 1.25984 & 0.162167 \\
\hline
\end{tabular}

Table 2 A parameter $\alpha$ estimation and estimate the average mean square error (AM) of function (1) the second part of the estimation methods based on simulation results for different values $(\alpha, \beta, \gamma, \theta)$ of EWG distribution
Tables 1, 2, 3, 4 show that the BSPE and the BTPE are the best. On the other hand based on a 1000 random samples simulated from the EWG distribution, Fig. 1 show the graphs of estimations of the pdf (1) for the estimation methods of the third section which is given in Table 5, which represents the superiority of the BTPE toward other estimates. 
Table 3 parameters $\gamma$ and $\beta$ estimation and estimate the average mean square error (AM) of function (2) the second part of the estimation methods based on simulation results for different values $(\alpha, \beta, \gamma, \theta)$ of EWG distribution

\begin{tabular}{|c|c|c|c|c|c|c|c|c|c|c|}
\hline$(\alpha, \beta, \gamma, \theta)$ & $n$ & $\hat{\gamma}_{B T P E}$ & $\hat{\beta}_{B T P E}$ & $\hat{\gamma}_{P C E}$ & $\hat{\beta}_{P C E}$ & $\hat{\gamma}_{M L E}$ & $\hat{\beta}_{P C E}$ & $A M\left(\hat{F}_{M L E}\right)$ & $A M\left(\hat{F}_{P C E}\right)$ & $A M\left(\hat{F}_{B T P E}\right)$ \\
\hline \multirow[t]{5}{*}{$(2,3,4.5,0.6)$} & 100 & 4.8367 & 1.0079 & 1.4786 & 2.2954 & 1.9731 & 3.6372 & 0.15101 & 0.13021 & 0.03613 \\
\hline & 200 & 4.6467 & 1.0102 & 1.4728 & 2.2611 & 1.9642 & 3.6005 & 0.15825 & 0.07952 & 0.03390 \\
\hline & 300 & 4.5511 & 1.0110 & 1.4551 & 2.2551 & 1.9634 & 3.5936 & 0.15983 & 0.08319 & 0.03276 \\
\hline & 400 & 4.6769 & 1.0109 & 1.4498 & 2.2295 & 1.9586 & 3.5841 & 0.15672 & 0.08829 & 0.03338 \\
\hline & 500 & 4.6228 & 1.0108 & 1.4518 & 2.2351 & 1.9594 & 3.5835 & 0.15933 & 0.08519 & 0.03356 \\
\hline \multirow[t]{5}{*}{$(4,1.5,3,0.8)$} & 100 & 4.8612 & 1.1072 & 2.4553 & 0.8936 & 0.3301 & 1.1672 & 0.05273 & 0.07924 & 0.03038 \\
\hline & 200 & 4.2922 & 1.1492 & 2.4476 & 0.8332 & 0.3299 & 1.1652 & 0.03054 & 0.05521 & 0.02974 \\
\hline & 300 & 4.0555 & 1.1412 & 2.4444 & 0.7956 & 0.3295 & 1.1652 & 0.02117 & 0.03104 & 0.01774 \\
\hline & 400 & 3.8799 & 1.1577 & 2.4409 & 0.7693 & 0.3292 & 1.1656 & 0.02989 & 0.04122 & 0.01507 \\
\hline & 500 & 3.7940 & 1.1510 & 2.4426 & 0.7542 & 0.3293 & 1.1651 & 0.02296 & 0.10221 & 0.00343 \\
\hline \multirow[t]{5}{*}{$(1.5,0.25,2,0.2)$} & 100 & 2.0932 & 1.5782 & 0.1531 & 1.6962 & 2.5424 & 0.2540 & 0.22450 & 0.09921 & 0.00272 \\
\hline & 200 & 2.0281 & 1.5801 & 0.1093 & 3.0855 & 2.5072 & 0.2534 & 0.22421 & 0.09616 & 0.00240 \\
\hline & 300 & 2.0289 & 1.5799 & 0.0902 & 6.4183 & 2.4900 & 0.2531 & 0.22577 & 0.09368 & 0.00213 \\
\hline & 400 & 2.0029 & 1.5769 & 0.0783 & 24.016 & 2.4718 & 0.2526 & 0.2258 & 0.09434 & 0.00215 \\
\hline & 500 & 2.0118 & 1.5771 & 0.0696 & 11.233 & 2.4811 & 0.2529 & 0.22661 & 0.09229 & 0.01925 \\
\hline \multirow[t]{5}{*}{$(2,3.5,4,0.5)$} & 100 & 4.1289 & 1.0064 & 0.9515 & 2.7688 & 2.3723 & 3.8177 & 0.18144 & 0.07928 & 0.01769 \\
\hline & 200 & 4.1988 & 1.0113 & 0.9424 & 2.7571 & 2.3536 & 3.8049 & 0.17706 & 0.10130 & 0.01735 \\
\hline & 300 & 4.0719 & 1.0101 & 0.9372 & 2.7964 & 2.3449 & 3.7993 & 0.18281 & 0.08311 & 0.01692 \\
\hline & 400 & 4.1220 & 1.0111 & 0.9341 & 2.8412 & 2.3407 & 3.7961 & 0.17867 & 0.08771 & 0.01699 \\
\hline & 500 & 4.0517 & 1.0105 & 0.9313 & 2.7955 & 2.3429 & 3.7972 & 0.18344 & 0.09134 & 0.01726 \\
\hline
\end{tabular}

Table 4 parameters $\alpha$ and $\beta$ estimation and estimate the average mean square error (AM) of function (1) the second part of the estimation methods based on simulation results for different values $(\alpha, \beta, \gamma, \theta)$ of EWG distribution

\begin{tabular}{|c|c|c|c|c|c|c|c|c|c|c|}
\hline$(\alpha, \beta, \gamma, \theta)$ & $n$ & $\hat{\gamma}_{B T P E}$ & $\hat{\beta}_{B T P E}$ & $\hat{\gamma}_{P C E}$ & $\hat{\beta}_{P C E}$ & $\hat{\gamma}_{M L E}$ & $\hat{\beta}_{P C E}$ & $A M\left(\hat{f}_{M L E}\right)$ & $A M\left(\hat{f}_{P C E}\right)$ & $A M\left(\hat{f}_{B T P E}\right)$ \\
\hline \multirow[t]{5}{*}{$(2,3,4.5,0.6)$} & 100 & 4.8367 & 1.0079 & 1.4786 & 2.2954 & 1.9731 & 3.6372 & 0.77035 & 4.34874 & 0.48280 \\
\hline & 200 & 4.6467 & 1.0102 & 1.4728 & 2.2611 & 1.9642 & 3.6005 & 0.79027 & 4.46875 & 0.43818 \\
\hline & 300 & 4.5511 & 1.0110 & 1.4551 & 2.2551 & 1.9634 & 3.5936 & 0.78236 & 4.48392 & 0.46429 \\
\hline & 400 & 4.6769 & 1.0109 & 1.4498 & 2.2295 & 1.9586 & 3.5841 & 0.78222 & 4.42212 & 0.46307 \\
\hline & 500 & 4.6228 & 1.0108 & 1.4518 & 2.2351 & 1.9594 & 3.5835 & 0.77847 & 4.47561 & 0.48618 \\
\hline \multirow[t]{5}{*}{$(4,1.5,3,0.8)$} & 100 & 4.8612 & 1.1072 & 2.4553 & 0.8936 & 0.3301 & 1.1672 & 0.02392 & 0.00994 & 0.00931 \\
\hline & 200 & 4.2922 & 1.1492 & 2.4476 & 0.8332 & 0.3299 & 1.1652 & 0.02798 & 0.01020 & 0.00859 \\
\hline & 300 & 4.0555 & 1.1412 & 2.4444 & 0.7956 & 0.3295 & 1.1652 & 0.02561 & 0.01051 & 0.00859 \\
\hline & 400 & 3.8799 & 1.1577 & 2.4409 & 0.7693 & 0.3292 & 1.1656 & 0.02709 & 0.01077 & 0.00793 \\
\hline & 500 & 3.7940 & 1.1510 & 2.4426 & 0.7542 & 0.3293 & 1.1651 & 0.02778 & 0.01095 & 0.00795 \\
\hline \multirow[t]{5}{*}{$(1.5,0.25,2,0.2)$} & 100 & 2.0932 & 1.5782 & 0.1531 & 1.6962 & 2.5424 & 0.2540 & 3.47621 & 0.01412 & 0.00817 \\
\hline & 200 & 2.0281 & 1.5801 & 0.1093 & 3.0855 & 2.5072 & 0.2534 & 3.14124 & 0.00890 & 0.00808 \\
\hline & 300 & 2.0289 & 1.5799 & 0.0902 & 6.4183 & 2.4900 & 0.2531 & 2.65151 & 0.00786 & 0.00745 \\
\hline & 400 & 2.0029 & 1.5769 & 0.0783 & 24.016 & 2.4718 & 0.2526 & 2.53194 & 0.00866 & 0.00816 \\
\hline & 500 & 2.0118 & 1.5771 & 0.0696 & 11.233 & 2.4811 & 0.2529 & 2.28138 & 0.00862 & 0.00798 \\
\hline \multirow[t]{5}{*}{$(2,3.5,4,0.5)$} & 100 & 4.1289 & 1.0064 & 0.9515 & 2.7688 & 2.3723 & 3.8177 & 1.87422 & 0.80433 & 0.12703 \\
\hline & 200 & 4.1988 & 1.0113 & 0.9424 & 2.7571 & 2.3536 & 3.8049 & 1.89349 & 0.70437 & 0.00981 \\
\hline & 300 & 4.0719 & 1.0101 & 0.9372 & 2.7964 & 2.3449 & 3.7993 & 1.84600 & 0.60702 & 0.06714 \\
\hline & 400 & 4.1220 & 1.0111 & 0.9341 & 2.8412 & 2.3407 & 3.7961 & 1.87497 & 0.62749 & 0.95869 \\
\hline & 500 & 4.0517 & 1.0105 & 0.9313 & 2.7955 & 2.3429 & 3.7972 & 1.89357 & 0.65273 & 0.92011 \\
\hline
\end{tabular}




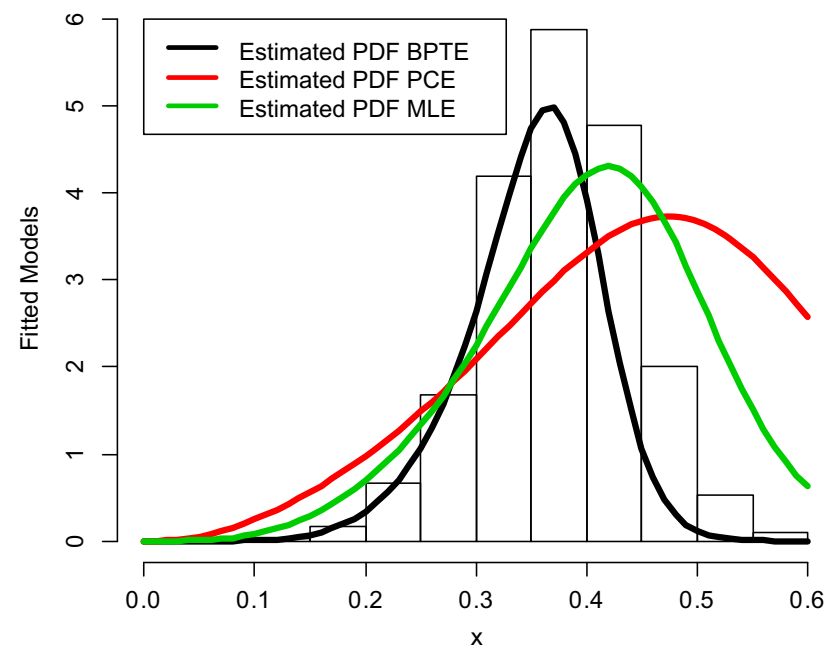

Fig. 1 The graphs of estimations BTPE, PCE and MLE of the pdf (1)

Table 5 Estimate of parameters and corresponding log-liklihood

\begin{tabular}{llll}
\hline & Estimate of $\gamma$ & Estimate of $\beta$ & Log-liklihood \\
\hline BTPE & 3.2728787 & 3.7482284 & -789.0882 \\
PCE & 2.9071186 & 2.8885803 & -797.1267 \\
MLE & 2.7120806 & 3.5376112 & -805.6754 \\
\hline
\end{tabular}

\subsection{Application with real data set}

In this section the BSPE, BTPE, PCE and MLE of pdf and cdf for the EWG distribution are computed and compared for a real data. The data is the waiting times (in minutes) of 100 bank customers collected by Ghitany et al. (2008) presented in the 'Appendix'. For known parameters $\beta=5.16, \gamma=0.55, \theta=0.95$ based on MLE method, Table 6 shows the average (AV) and corresponding mean square error (MSE) of the BSPE, PCE, MLE of pdf (1), cdf (2). Comparing theses results show that the BSPE provides better fit to waiting time data.

Also, For known parameters $\alpha=2.11, \theta=0.85$ based on MLE method, Table 7 shows the average (AV) and corresponding mean square error (MSE) of the BSPE, PCE, MLE of pdf (1), cdf (2). Comparing theses results show that the BTPE provides better fit to waiting time data.

\section{Conclusion}

In this research, the pdf and the cdf of the four-parameter EWG distribution were determined using several methods. To do this task, we first assume for an unknown parameter the BSPE, PCE and MLE of these functions are obtained. Then for two unknown parameters the BTPE, PCE and MLE of these functions are found. Then Using the Monte
Table 6 Estimate the average (AV) estimation and corresponding mean square error of pdf (1) and cdf (2)

\begin{tabular}{lllll}
\hline Method & AV (f) & MSE (f) & AV (F) & MSE (F) \\
\hline BSPE & 0.06834893 & 0.05809714 & 0.8869903 & 0.2006903 \\
MLE & 0.07025214 & 0.07020127 & 0.7384733 & 0.3131502 \\
PCE & 0.05329464 & 0.07918471 & 0.6097365 & 0.3685202 \\
\hline
\end{tabular}

Table 7 Estimate the average (AV) estimation and corresponding mean square error of pdf (1) and cdf (2)

\begin{tabular}{lllll}
\hline Method & AV (f) & MSE (f) & AV (F) & MSE (F) \\
\hline BTPE & 0.01335107 & 0.00393291 & 0.1659099 & 0.0798941 \\
MLE & 0.03688957 & 0.08424045 & 0.9484732 & 0.1200633 \\
PCE & 0.03862066 & 0.12865875 & 0.9691315 & 0.1256156 \\
\hline
\end{tabular}

Carlo simulation and real data set, it was shown that the BSPE and BTPE are better than the other estimators.

Open Access This article is distributed under the terms of the Creative Commons Attribution 4.0 International License (http://crea tivecommons.org/licenses/by/4.0/), which permits unrestricted use, distribution, and reproduction in any medium, provided you give appropriate credit to the original author(s) and the source, provide a link to the Creative Commons license, and indicate if changes were made.

\section{Appendix}

The waiting times (in minutes) of 100 bank customers

\footnotetext{
$0.8,0.8,1.3,1.5,1.8,1.9,1.9,2.1,2.6,2.7,2.9,3.1,3.2,3.3,3.5,3.6$, $4.0,4.1,4.2,4.2,4.3,4.3,4.4,4.4,4.6,4.7,4.7,4.8,4.9,4.9,5.0$, $5.3,5.5,5.7,5.7,6.1,6.2,6.2,6.2,6.3,6.7,6.9,7.1,7.1,7.1,7.1$, $7.4,7.6,7.7,8.0,8.2,8.6,8.6,8.6,8.8,8.8,8.9,8.9,9.5,9.6,9.7$, $9.8,10.7,10.9,11.0,11.0,11.1,11.2,11.2,11.5,11.9,12.4,12.5$, $12.9,13.0,13.1,13.3,13.6,13.7,13.9,14.1,15.4,15.4,17.3,17.3$, $18.1,18.2,18.4,18.9,19.0,19.9,20.6,21.3,21.4,21.9,23.0,27.0$, $31.6,33.1,38.5$.
}

\section{References}

Alizadeh M, Bagheri SF, Kaleghy Mogaddam M (2013) Efficient estimation of the density and cumulative distribution function of the generalized Rayleigh distribution. J Stat Res Iran 10(1):1-22

Bagheri SF, Alizadeh M, Baloui Jamkhaneh E, Nadarajah S (2013a) Evaluation and comparison of estimations in the generalized exponential-Poisson distribution. J Stat Comput Simul. https:// doi.org/10.1080/00949655.2013.793342

Bagheri SF, Alizadeh M, Nadarajah S (2013b) Efficient estimation of the PDF and the CDF of the exponentiated Gumbel distribution. Commun Stat Simul Comput 45(1):339-361 
Dixit UJ, Jabbari Nooghabi M (2010) Efficient estimation in the Pareto distribution. Stat Methodol 7:687-691

Dubey SD (1967) Some percentile estimators for Weibull parameters. Technometrics 9:119-129

Ghitany ME, Nadarajah S, Atieh B (2008) Lindley distribution and its application. Math Comput Simul 78(4):493-506

Jabbari Nooghabi M, Jabbari Nooghabi H (2010) Efficient estimation of pdf, CDF and rth moment for the exponentiated Pareto distribution in the presence of outliers. Statistics 44:1-20

Johnson NL, Kotz S, Balakrishnan N (1994) Continuous univariate distribution, vol 1, 2nd edn. Wiley, New York

Kao JHK (1958) Computer methods for estimating Weibull parameters in reliability studies. Trans IRE Reliab Qual Control $13: 15-22$
Kao JHK (1959) A graphical estimation of mixed Weibull parameters in life testing electrontubes. Technometrics 1:389-407

Mahmoudi E, Shiran M (2012) Exponentiated Weibull-geometric distribution and its applications, http://arxiv.org/abs/1206. $4008 \mathrm{v} 1$

Menon MV (1963) Estimation of the shape and scale parameters of the Weibull distribution. Technometrics 5:175-182

Zanakis Stelios H, Mann Nancy R (1982) A good simple percentile estimator of the Weibull shape parameter for use when all three parameters are unknown. Naval Res Logist 29:419-428 